\title{
Congestive heart failure caused by myxomatous mitral valve degeneration in a Siberian tiger
}

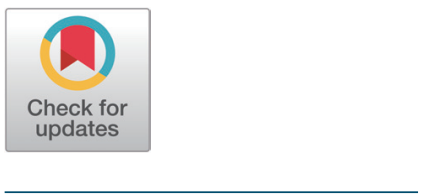

Received: Sep 23, 2021

Revised: Sep 24, 2021

Accepted: Sep 28, 2021

*Corresponding author

Byeongwoo Ahn

Laboratory of Veterinary Pathology,

College of Veterinary Medicine,

Chungbuk National University,

Cheongju 28644, Korea

Tel: +82-43-261-2508

E-mail: bwahn@cbu.ac.kr

Copyright $\odot 2021$ Research Institute of Veterinary Medicine, Chungbuk National University. This is an Open Access article distributed under the terms of the Creative Commons Attribution Non-Commercial License (http://creativecommons.org/licenses/ by-nc/4.0/) which permits unrestricted non-commercial use, distribution, and reproduction in any medium, provided the original work is properly cited.

\section{ORCID}

Jinsung Park

https://orcid.org/0000-0002-6942-4392 Jong-uk Kim

https://orcid.org/0000-0002-8229-0114 Jeongho Kim

https://orcid.org/0000-0002-8562-6306

Gonhyung Kim

https://orcid.org/0000-0002-9384-4741

Byeongwoo Ahn

https://orcid.org/0000-0002-4112-7724

\section{Conflict of Interest}

No potential conflict of interest relevant to this article was reported.

\section{Acknowledgements}

This research was supported by Chungbuk National University Korea National University Development project (2020)

\section{Ethics Approva}

This article does not require IRB/ IACUC approval because there are no

human and animal participants.

\author{
Jinsung Park ${ }^{1}$, Jong-uk Kim¹, Jeongho Kim², Gonhyung Kim³, \\ Byeongwoo Ahn ${ }^{1 *}$ \\ ${ }^{1}$ Laboratory of Veterinary Pathology, College of Veterinary Medicine, Chungbuk National University, \\ Cheongju 28644, Korea \\ ${ }^{2}$ Cheongju Zoo, Cheongju 28311, Korea \\ ${ }^{3}$ Laboratory of Veterinary Surgery and Veterinary Teaching Hospital, College of Veterinary Medicine, \\ Chungbuk National University, Cheongju 28644, Korea
}

\begin{abstract}
A 19-year-old male Siberian tiger was presented with inappetence and paralysis of hind limbs. In a computed tomography (CT) scan, intervertebral disk disease at L3-L4 was detected. Cardiac arrest occurred during the surgery. At autopsy, myxomatous mitral valve degeneration (MMVD) and eccentric hypertrophy of the left heart were noted. The diagnosis was congestive heart failure caused by MMVD. Microscopically, myocardial and pulmonary fibrosis were observed in addition to the disintegration of the fibrosa layer and accumulation of glycosaminoglycans and proteoglycans in the spongiosa layer of the mitral valve. This is the first case of congestive heart failure with MMVD in a Siberian tiger.
\end{abstract}

Keywords: congestive heart failure, myxomatous mitral valve degeneration, tiger

\section{INTRODUCTION}

Myxomatous valvular degeneration (MVD) or valvular endocardiosis is one of the most common acquired cardiac diseases in dogs. Although it can occur in any valves, $84 \%$ of the MVD is found at the mitral valve at autopsies [1]. The lesion in the mitral valve is thus commonly referred to as myxomatous mitral valve degeneration (MMVD). MMVD is highly correlated with ages and progresses slowly. Male dogs are more susceptible to MMVD with earlier development and clinical significances [2]. Although MMVD can affect all canine breeds, the high prevalence is shown in small to medium-sized breeds [2]. Especially, such breeds as Cavalier King Charles Spaniels (CKCS) and Dachshunds are more susceptible to MMVD and present early development and clinical severity [3, 4]. However, Labrador Retrievers and German Shepherds seem to be resistant to MMVD [5]. The progression of mitral valve prolapse (MVP) in human has been described to be very similar to that of MMVD in dogs. Some of the similarities between MMVD and MVP include their slow progression, gross and microscopic lesions, correlation with age and gender and polygenic inheritance [6]. The prevalence of MMVD in cats has rarely been studied, 
but is believed to be low [7]. MVD has been reported in pigs and horses, but not in wild felids [8, 9].

Although the etiology of MMVD is rarely known, inheritance is believed to be highly associated. CKCS and Dachshunds show polygenic inheritance of MMVD [7]. The pathogenesis of the myxomatous degeneration is rarely known. However, it has been suggested that endothelial damages of the affected valve are associated with subsequent changes in valvular interstitial cells.

MVD leads to thickening and shortening of the valve leaflets. The deformity of valve leaflets causes primary mitral regurgitation, easily tolerated by the left atrium without a significant decrease of the stroke volume [7]. As the MVD progresses, the regurgitant volume into the left atrium increases, and hence the ventricular stroke volume decreases. Cardiac and neurohormonal compensatory mechanism works to maintain the cardiac output. The dilation of the left atrium compensates the increased regurgitant volume and prevents the increase of intra-atrial and pulmonary venous pressure. However, subsequent left atrial and ventricular dilation lead to abnormal apposition of the leaflets, thus resulting in secondary mitral regurgitation [7]. When the mitral regurgitation reaches over the threshold of cardiac compensation, pulmonary congestion and edema may occur. In slowly progressing MMVD, the dilatation of the left atrium compensates the regurgitant volume, and pulmonary congestion and edema may develop later. Volume overload also induces eccentric hypertrophy to accommodate the increased end diastolic volume in the left ventricle. Initial hypertrophy aids the maintenance of the ventricular stroke volume. However, further progression of the hypertrophy leads to myocardial fibrosis, causing the impairment of myocardial contractibility. It has been suggested that such myocardial hypertrophy is induced by increased concentration of angiotensin II in the myocardium.

The pathology of the MVD has been widely studied in dogs. The free edges of the leaflets, where primary chordae tendinae are attached, are most frequently affected by myxomatous degeneration [10]. The edges become thickened and opaque, and prolapse toward the left atrium. When the primary chordae tendinae are ruptured, mitral regurgitation may be aggravated acutely, leading to congestive heart failure (CHF) [10]. According to Whitney, the valvular lesions can be classified into four types based on the morphological features. Type I (minimal): A few, discrete nodules in contact areas with irregular areas of opacity; Type II (mild), multiple large nodules in contact areas with further increased opacity; Type III (moderate), large coalescing nodules forming plaque-like deformities with chordae tendinae thickened; Type IV (severe), gross distortions of the valve by coalescing nodules and plaques $[1,10,11]$. The valve leaflet consists of 4 layers: the ventricularis, the fibrosa, the spongiosa and the atrialis layer. The ventricularis and atrialis layer consist of the endothelium and subendothelial layer facing the respective aspects. The spongiosa layer is loose connective tissue with interstitial cells. The fibrosa layer consists of dense collagen tissue which elongates into the chordae tendinae. The most prominent histopathological feature is disintegration of the fibrosa layer and accumulation of glycosaminoglycans in the spongiosa layer $[5,10]$. The accumulation of glycosaminoglycans leads to ballooning of the leaflet [10]. Damages and loss of the endothelial 
layers are often observed microscopically.

In this report, we describe the gross and histopathological characteristics of MMVD occurred in a Siberian tiger.

\section{CASE}

A 19-year-old male Siberian tiger was referred to Veterinary Teaching Hospital, Chungbuk National University with a history of inappetence, lethargy and paralysis of hind limbs. In blood analysis, serum creatinine level increased to $3.3 \mathrm{mg} / \mathrm{dL}$. Computed tomography (CT) scan showed intervertebral disk disease at the L3-L4 and osteoarthritis of the spine. The case died of cardiac arrest during the surgery for the intervertebral disk disease. At autopsy, the pericardium was moderately filled with serosanguineous fluid (Fig. 1A). The mitral valve was generally thickened with some nodules at the distal end of the leaflets (Fig. 1B). One of the chordae tendinae was ruptured and disconnected from the anterior mitral valve leaflet. The endocardium of the left atrium was opaque and thickened. The lungs were diffusely congested in dark-red tone. On the surface of the lungs, there were multifocal inflated areas in balloon-like appearances.

The lumbar spinal cord between the L3 and L4 was severely compressed by the protruded intervertebral disk below (Fig. 1C). The peritoneal cavity was moderately filled with red-col-
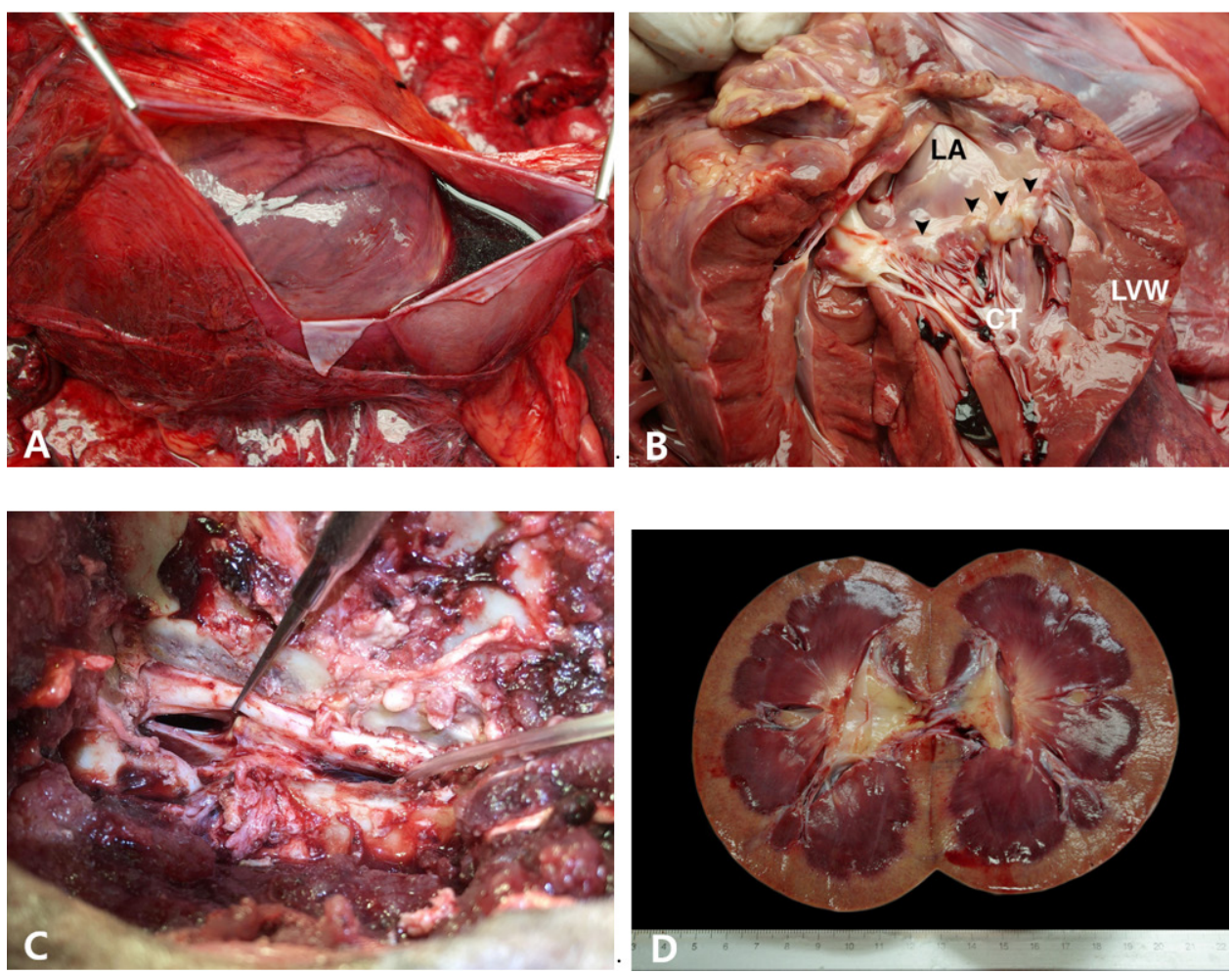

Fig. 1. Gross findings. (A) The pericardium filled with a large amount of serosanguineous fluid. (B) The posterior aspect of the left ventricle. Myxomatous degeneration of the posterior valve leaflets presents Whitney type III (arrowheads). (C) Severe intervertebral disk herniation at the L3-L4. (D) Renal cortex with petechiae and severely congested medulla. PC, pericardium; LA, left atrium; LVW, left ventricular wall; CT, chordae tendineae. 
ored, watery fluid. The kidney was generally swollen. The cortex was generally pale with numerous petechiae scattered. The medulla was diffusely congested in magenta tone (Fig. 1D). The urinary bladder was distended like a balloon and filled with deep-honey-colored, clear watery fluid mixed with opaque sulfur-yellow, watery fluid. The fluid reached up to 4L in total. The right lobe of the thyroid gland was replaced with multifocal cysts containing a moderate amount of clear fluid. There was a circular, $1 \mathrm{~cm}$ in diameter ulcer in the body of the stomach. One of the testes was placed in the retroperitoneum in a bumpy appearance of the surface. The size of the undescended testis was much smaller than the other testis in the scrotum and intensely firm in consistency.

Tissues samples were fixed in 10\% phosphate-buffered formalin, processed and embedded in paraffin for histopathological examinations. Four-micrometer-thick tissue sections were used for staining with hematoxylin and eosin, Masson's trichrome stain and alcian-blue stain.

For Masson's trichrome stain, sections were deparaffinized and rehydrated and refixed in Bouin's solution (Sigma-Aldrich, St. Louis, MO, USA) for 24 hours. Then, the sections were stained in Weigert's iron hematoxylin solution (Polysciences, Warrington, PA, USA) and Biebrich-scarlet fuschin solution (Polysciences) for 10 minutes, respectively. They were differentiated in phosphomolybdic-phosphotungstic acid (Fluka Chemical Co., Hauppauge, NY) and incubated in aniline blue solution (Polysciences Inc., Warrington, PA). For visualization of
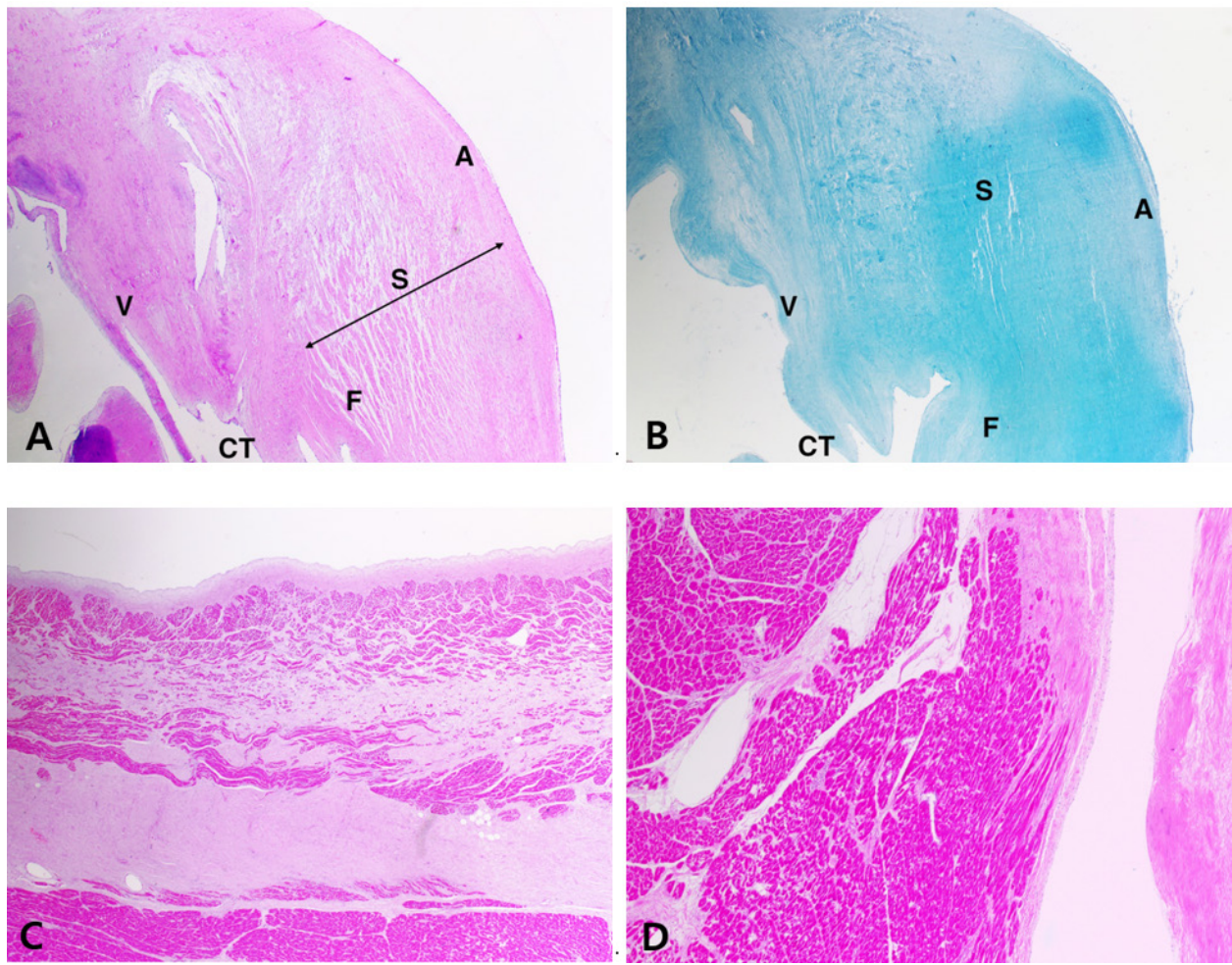

Fig. 2. Microscopic findings of the heart wall and mitral valve leaflet. Note the myxomatous thickening of the cusp of the mitral valve. (A) The spongiosa layer (S) is severely extended and the fibrosa layer (F) is disintegrated by infiltration of glycosaminoglycans and proteoglycans (HE $\times 25)$. (B) The deposition of the glycosaminoglycans in the mitral valve leaflet is highlighted in light blue (Alcian blue $\times 25$ ). (C) In the left atrium wall, note the severely proliferated fibrous tissues in subendocardium and atrophied myocardium $(H E \times 25)$. (D) Myocardial fibrosis in the subendocardium of the left ventricle $(H E \times 100) . A$, atrialis layer; $\mathrm{S}$, spongiosa layer; $\mathrm{F}$, fibrosa layer; $\mathrm{V}$, ventricularis layer. 
mucin, alcian blue and nuclear fast red solution were purchased from Sigma-Aldrich company and the staining procedure was performed according to the manufacturer's guides.

Histologically, the mitral valve showed the typical lesion of MVD with general thickening of the leaflet in a balloon-like appearance. The fibrosa layer has been disintegrated and replaced with mucoid substances. The spongiosa layer is extended by an excessive accumulation of extracellular matrix (Fig. 2A). The accumulation of glycosaminoglycan was seen in alcian-blue stain (Fig. 2B). In the left atrial wall, a band of subendocardial fibrosis was extensive with myocardial atrophy (Fig. 2C). The left ventricle had multifocal areas of myocardial atrophy and fibrosis. The myocardial fibers were edematous and loosened with proliferation of connective tissue (Fig. 2D). Lung also showed chronic lesions characterized by loss of normal alveolar architecture with severe thickening of alveolar walls by fibrosis (Fig. 3A and B). The kidney was undergoing chronic nephropathy with tubulointerstitial nephritis characterized by tubular necrosis, glomerular atrophy, protein casts and interstitial lymphocytic infiltration (Fig. $3 \mathrm{C}$ and D).

\section{DISCUSSION}

The lesion of MMVD had been classified into four types as described previously. Myxomatous degeneration of the mitral valve in this case is considered mostly Whitney type III [1,
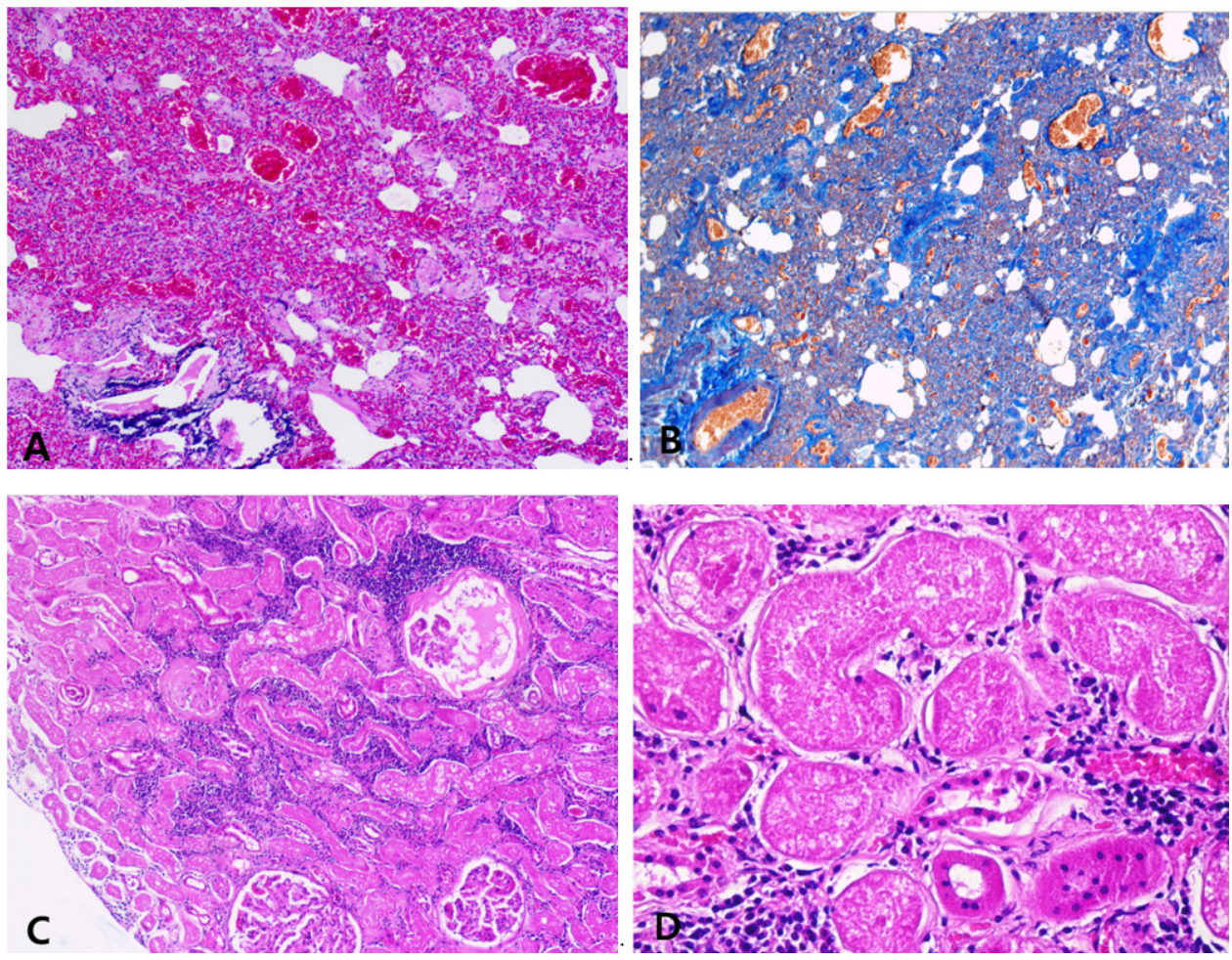

Fig. 3. Microscopic findings of lung and kidney. (A) Chronic pulmonary lesion. Note that most alveoli are compressed by thickened interstitial tissue, and alveolar capillaries are severely congested (HE $\times 100$ ). (B) Areas stained in blue indicate the proliferated fibrous tissue around the damaged alveoli (Masson's trichrome $\times 40$ ). (C) Severe nephropathy. Note the diffuse tubular necrosis with infiltration of inflammatory cells. (HE × 100). (D) High power view of C (HE × 400). 
10, 11]. Large nodules were found at the distal end of the mitral valve leaflets. The anterior mitral valve leaflet seems more degenerative than the posterior one. The anterior leaflet presents hemorrhage and rupture of a primary chorda tendina. It is known that the anterior leaflet is more susceptible to mechanical stresses due to its higher proportion and mobility, leading to more aggressive degeneration [7]. The rupture of the primary chorda tendina in the anterior leaflet is assumed to have occurred acutely and aggravated mitral regurgitation. Pericardial effusion may have been caused by CHF and developed into cardiac tamponade, which further reduced the myocardial contractility. The layers of the mitral valve have become indistinguishable with the proliferation of extracellular matrix. The spongiosa layer is thickened by the accumulation of glycosaminoglycans and proteoglycans, whereas the fibrosa layer has lost its integrity. The gross appearance of the left atrium was opaque and thickened as a result of extensive subendocardial fibrosis. Initial eccentric hypertrophy may have compensated the increased intra-atrial volume overload. However, further progression of the valvular disease has led to ischemia, necrosis and fibrosis of myocardium. Myocardial fibrosis in the left ventricle is another evidence of cardiac remodeling and eccentric hypertrophy. In this case, the cardiac remodeling has not been progressed as much as the case of the dog with MMVD inheritance. Thus, pulmonary congestion and edema may have developed earlier, further leading to severe necrosis and fibrosis of the alveolar wall. It is difficult to point out clinical signs of a wildlife or captive animal, but it is assumed that the tiger had suffered from severe respiratory distress. These cardiopulmonary conditions may have been aggravated by general anesthesia for surgery of intervertebral disk disease.

This is the first report of CHF caused by MMVD in the tiger. Because of the difference in anatomical structure and physiological features between the dog and the tiger, it is difficult to assess the precise grade of the disease. However, similar morphological and histological features of MMVD has been found in this study, and it is believed to share similar pathophysiology.

According to several retrospective studies, it is known that such diseases as chronic kidney disease, intervertebral disk disease, infective pulmonary diseases and other neoplasia are common causes of death in wild felids [12-14]. Especially, chronic kidney disease with tubulointerstitial nephritis is characteristically observed in postmortem examination of tigers [13]. Severe nephropathy with tubulointerstitial nephritis found in histological examination is in accordance with the precedent retrospective studies. The increased concentration of serum creatinine $(3.3 \mathrm{mg} / \mathrm{dL})$ reflects moderate to severe azotemia. This renal lesion may not have been the primary cause of death, but it may have aggravated the general health status of the tiger.

\section{REFERENCES}

1. Zachary JF. Mechanisms of microbial infections. In: Zachary JF (ed.). Pathologic basis of veterinary diseases. 6th ed. St. Louis: Elsevier; 2017.

2. Thrusfield MV, Aitken CGG, Darker PGG. Observations on breed and sex in relation to canine heart valve incompetence. J Small Anim Pract 1985;26:709-717. 
3. Häggström J, Hansson K, Kvart C, Swenson L. Chronic valvular disease in the cavalier King Charles spaniel in Sweden. Vet Rec 1992;131:549-553.

4. Olsen LH, Fredholm M, Pedersen HD. Epidemiology and inheritance of mitral valve prolapse in Dachshunds. J Vet Intern Med 1999;13:448-456.

5. Maxie GM. Jubb KVF. Kennedy and Palmer's pathology of domestic animals. 6th ed. St. Louis: Elsevier; 2016.

6. Pedersen HD, Häggström J. Mitral valve prolapse in the dog: a model of mitral valve prolapse in man. Cardiovasc Res 2000;47:234-243.

7. Ettinger SJ, Feldman EC, Cote E. Textbook of veterinary internal medicine. 8th ed. St. Louis: Elsevier; 2017.

8. Gagna C, Meier D, Ru G, Pospischil A, Guarda F. Pathology of mitral valve in regularly slaughtered pigs: an abattoir survey on the occurrence of myxoid degeneration (endocardiosis), fibrosis and valvulitis. Zentralbl Veterinarmed A 1998;45:383-395.

9. Reef VB, Bain FT, Spencer PA. Severe mitral regurgitation in horses: clinical, echocardiographic and pathological findings. Equine Vet J 1998;30:18-27.

10. Fox PR. Pathology of myxomatous mitral valve disease in the dog. J Vet Cardiol 2012;14:103126.

11. Whitney JC. Cardiovascular pathology. J Small Anim Pract 1967;8:459-465.

12. Junginger J, Hansmann F, Herder V, Lehmbecker A, Peters M, Beyerbach M, et al. Pathology in captive wild felids at German zoological gardens. PLOS ONE 2015;10:e0130573.

13. Newkirk KM, Newman SJ, White LA, Rohrbach BW, Ramsay EC. Renal lesions of nondomestic felids. Vet Pathol 2011;48:698-705.

14. Owston MA, Ramsay EC, Rotstein DS. Neoplasia in felids at the Knoxville zoological gardens, 1979-2003. J Zoo Wildl Med 2008;39:608-613. 